\title{
Ultra-short and ultra-intense $x$-ray free-electron laser single pulse in one-dimensional photonic crystals
}

\author{
Jean-Michel André and Philippe Jonnard
}

Sorbonne Universités, UPMC Univ Paris 06, Laboratoire de Chimie Physique-Matière et Rayonnement, 11 rue Pierre et Marie Curie, F-75231 Paris cedex 05, France

CNRS UMR 7614, Laboratoire de Chimie Physique-Matière et Rayonnement, 11 rue Pierre et Marie Curie, F-75231 Paris cedex 05, France

\begin{abstract}
The propagation within a one-dimensional photonic crystal of a single ultrashort and ultra-intense pulse delivered by an x-ray free-electron laser is analysed with the framework of the time-dependent coupled-wave theory in non-linear media. It is shown that the reflection and the transmission of an ultra-short pulse present a transient period conditioned by the extinction length and also the thickness of the structure for transmission. For ultra-intense pulses, non-linear effects are expected: they could give rise to numerous phenomena, bi-stability, self-induced transparency, gap solitons, switching, ..., which have been previously evidenced in the optical domain.
\end{abstract}

\section{Keywords}

X-ray free electron laser, one-dimensional photonic crystal, non linear processes 


\section{Introduction}

The purpose of this work is to analyse different aspects of the time-domain propagation of an ultra-intense and/or ultra-short single pulse delivered by an x-ray free-electron laser (X-FEL) within a Bragg structure. In the hard x-ray domain, the Bragg structures firstly implemented were the natural crystals and the steady-state (time independent) diffraction by crystals was studied in details within the framework of the so-called dynamical theory of diffraction (Authier, 2003). Since the seventies, synthetic Bragg crystals based on periodic multilayer stacks, sometimes called multilayer interferential mirrors (MIMs) have been developed for the soft-x-ray domain for which natural crystals were unusable; the time-independent diffraction by these multilayer structure have also been extensively studied (Pardo et al., 1988).

In this paper, we consider a MIM but some conclusions could be drawn in a similar way to the case of a natural crystal diffracting in the Bragg (reflection) or Laue (transmission) geometry where only one dimension is involved. Thus, hereafter we use the generic term, one-dimensional photonic crystal (1D-PC) to refer to the two types of Bragg structures crystal or MIM. The term distributed-feedback structure (DFB) has also been commonly used in the literature to refer to this kind of structure. Let us outline that the terminologies PC and DFB are rarely used for the x-ray range but are more common in the long wavelength domain (optical range, ...). Indeed most of the effects that we describe in this paper for the x-ray domain can be found in the whole electromagnetic spectrum and in particular the non-linear (NL) processes have been studied in details in the optical domain mainly in the context of optics of fibers and waveguide Bragg gratings, so that a large part of Section 4 is borrowed from the numerous studies published on these topics. The objective of the present work is mainly to inform the community of X-FEL users about some linear and NL effects that could be evidenced with X-FEL radiation pulses.

The X-FEL facilities are now able to deliver single ultra-intense and ultra-short pulses of coherent radiation; considering for instance FERMI facility, the photon beam parameters for FEL1 are $100 \mu \mathrm{J}$ per pulse with an estimated pulse length (FWHM) less than $150 \mathrm{fs}$. Ultra-short means that the duration of the pulse is less that the relaxation times of the media, ultra-intense means that non-linear optical effects are expected and finally coherence means that the photons in a pulse have fixed phase relationships forming a single mode. All these unique features (high brightness, short time duration, temporal and spatial coherence) open the way to the observation of coherent non-linear (NL) processes. The induced polarization $\boldsymbol{P}$ of the materials is given as a power-series expansion of the incident electric field $\boldsymbol{E}$, in cgs units,

$$
\boldsymbol{P}=4 \pi \sum_{i \geq 1} \chi^{(i)}|\boldsymbol{E}|^{i-1} \boldsymbol{E}
$$

$\chi^{(i)}$ being the susceptibility of the $i^{\text {th }}$ order. Generally, the even order terms are null for any centro-symmetric system so that the lowest order NL processes is of the third order. One among them, the most famous one is the Kerr effect for which the refractive index change is proportional to the square of the applied electric field or electric field intensity. Recently x-ray reflectivity enhancement in titanium has been reported (Bencivenga et al., 2014) by a team using ultrafast XUV radiation at the TIMEX endstation of the EIS beamline of FERMI X-FEL facility; the experimental results have been interpreted as a dependence of the plasma frequency with respect to the energy density $\overline{\mathrm{E}} \sim|\boldsymbol{E}|^{2}$ which leads in the framework of the Drude refractive index model to variation of reflection upon the deposited energy density. The dependence of the refractive index 
upon $\bar{E}$ can be regarded has a special case of Kerr effect known as refractive index intensity dependence which can give rise to numerous NL effects observed and expected especially in the low energy domain of the electromagnetic spectrum: self-focusing, selfphase modulation, spatial solitons (New, 2014), NL surface polaritons (Leung, 1985). Intensity-dependent refractive index is also at the origin, in Bragg structure, of various effects such as gap solitons (Chen \& Mills, 1987; Mills \& Trullinger, 1987; Martijn de Sterke \& Sipe, 1994) connected to self-induced transparency (Aceves \& Wabnitz, 1989), pulse compression in Bragg optical fiber (Winful, 1985), bi-stability in NL distributed feedback structure (Winful et al., 1979).

Time-dependent diffraction by Bragg structure have comparatively received poor attention: Chukhovskii and Förster have considered time-dependent diffraction by crystal (Chukhovskii \& Förster, 1995) and more recently their approach has been extended to one-dimensional photonic crystal (André \& Jonnard, 2015). Let us mention that the response of Bragg structures, MIMs (Ksenzov et al., 2008, 2009; Bushuev \& Samoylova, 2011) and crystals (Shastri et al., 2001; Bushuev, 2008) to X-FEL sources, have been treated by means of methods implemented for the frequency domain.

We lead our investigation in the framework of the time-dependent coupled-wave, TDCW, analysis extended to NL materials. In Section 2 we establish the system of coupled-wave equations taking into account the NL response of the media. In Section 3 we consider the low-intensity regime, LIR, where NL behaviour can be neglected; we show that the indicial response of a MIM displays a transient period determined by the extinction length of the Bragg structure in terms of reflection and by the extinction length combined with the thickness of the structure in terms of transmission. In Section 4 we consider the high-intensity regime, HIR, with NL effects first in the steadystate case where bi-stability can be envisaged, then in the time-dependent case where we consider the possibility to observe Bragg solitons associated with a kind of selfinduced transparency of the Bragg structure.

\section{Time-dependent coupled-wave theory in non-linear 1D-PC}

We consider the propagation of an ultra-short and ultra-intense (generally coherent) single pulse such as the one delivered by an X-FEL in a 1D-PC as shown in Figure 1. The figure also gives the geometry of the problem and some notations. We consider a periodic stack of $N$ bilayers. The bilayer is made up of a material a with dielectric susceptibility $\chi_{a}$ and material $\mathbf{b}$ with dielectric susceptibility $\chi_{b}$ with layer thickness $d_{a}=\gamma d$ and $d_{b}=(1-\gamma) d$ respectively. The incoming radiation with a wave vector $\boldsymbol{k}=\left(k_{x}, k_{z}\right)$ in the plane $(\mathrm{x}, \mathrm{z})$ strikes the multilayer structure under a glancing angle $\theta$. A Cartesian orthogonal reference frame $(\hat{\mathbf{x}}, \hat{\mathbf{y}}, \hat{\mathbf{z}})$ is used. $L$ is the total thickness of the stack equal to $N d$. The reciprocal vector $\boldsymbol{g}=\frac{2 \pi}{d} \hat{\mathbf{Z}}$ is orthogonal to the stratification planes. 


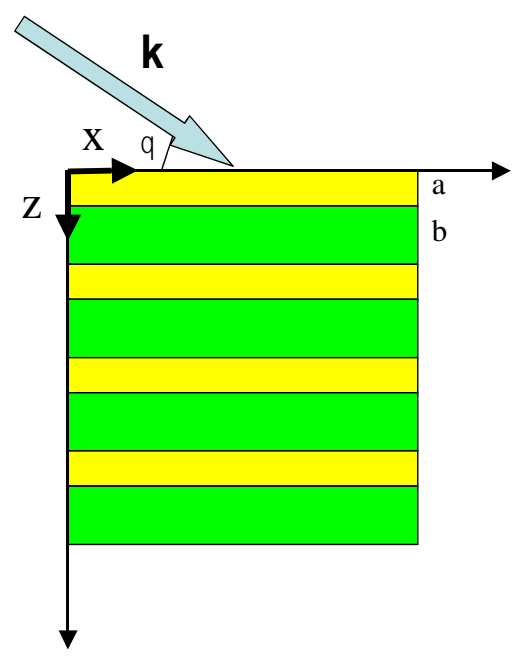

Figure 1: Sketch of the 1D-PC consisting in a periodic stack of alternating $\mathbf{a} / \mathbf{b}$ bilayers.

The electric field $\boldsymbol{E}(x, z, t)$ of the X-FEL single pulse is modelled by a quickly varying carrier with frequency $\omega=$ ? ? ? ? ? ? ? ? ? ? ? ? write it as follows (assuming a $s$-polarization case):

$$
\boldsymbol{E}(x, z, t)=E_{0}(z, t) e^{i\left(k_{x} x-\omega t\right)} \hat{\boldsymbol{y}}
$$

We assume that the polarization of the media follows instantly the change of the electric field but that the media have a NL behaviour; more precisely we consider that the medium is subject to the special case of optical Kerr effect for which the refractive index is intensity-dependent. Consequently we write the polarisation $\boldsymbol{P}$ as follows:

$$
\begin{gathered}
\boldsymbol{P}(x, z, t)=\chi\left(z, E_{0}(z, t)\right) \boldsymbol{E}(z, t) \\
=\left(\chi^{(1)}(z)+\chi^{(3)}(z)\left|E_{0}(z, t)\right|^{2}\right) E_{0}(z, t) e^{i\left(k_{x} x-\omega t\right)} \hat{\boldsymbol{y}}
\end{gathered}
$$

that corresponds to a third order NL. The susceptibilities $\chi^{(n)}(z)$ do not depend on time while the total susceptibility $\chi$ depends on time through $E_{0}(z, t)$, but they vary periodically along the $\mathrm{z}$ direction. Consequently they can be expanded in Fourier series:

$$
\chi^{(n)}(z)=\bar{\chi}^{(n)}+\sum_{p=-\infty}^{+\infty} \Delta \chi^{(n)} u_{p} e^{i p g z}
$$

with

$$
\begin{gathered}
\bar{\chi}^{(n)}=\chi_{a}^{(n)} \gamma+\chi_{b}^{(n)}(1-\gamma) \\
\Delta \chi^{(n)}=\chi_{a}^{(n)}-\chi_{b}^{(n)} \ll \bar{\chi}^{(n)} \\
u_{p}=\gamma \operatorname{sinc}(\pi p \gamma) e^{-i \pi p \gamma} \\
g=\frac{2 \pi}{d}
\end{gathered}
$$

In our study we restrict to the Kerr NL and consequently we keep only the terms $\bar{\chi}^{(1)}, \bar{\chi}^{(3)}, \Delta \chi^{(1)}$ and $\Delta \chi^{(3)}$. 
In the 1D-PC, the electric field envelope can be written as the superposition of two contra-propagating waves along the $z$-axis:

$$
E_{0}(z, t)=F(z, t) e^{+i \kappa z}+B(z, t) e^{-i \kappa z}
$$

with

$$
\kappa=k \sin \theta
$$

and using the following auxiliary amplitude terms

$$
\varepsilon_{+}(z, t)=F(z, t) \exp \left[-i\left(\frac{p g}{2}-\kappa\right) z\right]
$$

and

$$
\varepsilon_{-}(z, t)=B(z, t) \exp \left[+i\left(\frac{p g}{2}-\kappa\right) z\right]
$$

In the framework of the two-wave theory where only the $0^{\text {th }}$ order and the $p$ th Fourier term are strongly coupled (i.e. in the vicinity of the $p$ th Bragg resonance), it can be shown that in the slow-varying approximation both in space and in time, that the column amplitude vector $\bar{\varepsilon}(z, t)=\left(\begin{array}{l}\varepsilon_{+}(z, t) \\ \varepsilon_{-}(z, t)\end{array}\right)$ and its complex conjugate $\bar{\varepsilon}^{*}(z, t)=$ $\left(\begin{array}{l}\varepsilon_{+}{ }^{*}(z, t) \\ \varepsilon_{-}{ }^{*}(z, t)\end{array}\right)$ obey the following system of partial differential equations (PDEs) forming the so-called non-linear coupled mode equations with loss or gain:

$$
\partial_{z} \bar{\varepsilon}(z, t)=\overline{\mathcal{T}} \partial_{t} \bar{\varepsilon}(z, t)+i \overline{\mathcal{M}} \bar{\varepsilon}(z, t)+\overline{\mathcal{N}} \bar{\varepsilon}(z, t)+\overline{\mathcal{N}_{c}} \bar{\varepsilon}^{*}(z, t)
$$

where $\overline{\mathcal{M}}$ is the propagation matrix in space given by

$$
\overline{\mathcal{M}}=\left(\begin{array}{cc}
-\alpha & K^{+} \\
K^{-} & \alpha
\end{array}\right)
$$

with

$$
\begin{gathered}
\alpha=\frac{p g}{2}+\frac{k^{2}}{\kappa} 2 \pi \bar{\chi}^{(1)}-\kappa \\
K^{+}=-\frac{k^{2}}{\kappa} 2 \pi \Delta \chi^{(1)} u_{p} \\
K^{-}=\frac{k^{2}}{\kappa} 2 \pi \Delta \chi^{(1)} u_{-p}
\end{gathered}
$$

$\overline{\mathcal{T}}$ is the propagation matrix in time:

$$
\overline{\mathcal{T}}=\left(\begin{array}{cc}
-\frac{1}{c \sin \theta} & 0 \\
0 & \frac{1}{c \sin \theta}
\end{array}\right)
$$


$\overline{\mathcal{N}}$ is the NL matrix corresponding to the average NL term:

$$
\overline{\mathcal{N}}=\left(\begin{array}{ll}
-\Gamma_{a} \Lambda_{+}(z, t, S, X) & \Gamma_{p \Delta} \Lambda_{-}(z, t, S, X) \\
-\Gamma_{p \Delta} \Lambda_{+}(z, t, S, X) & \Gamma_{a} \Lambda_{-}(z, t, S, X)
\end{array}\right)
$$

with

$$
\begin{aligned}
& \Lambda_{+}(z, t, S, X)=S\left|\varepsilon_{+}(z, t)\right|^{2}+2 X\left|\varepsilon_{-}(z, t)\right|^{2} \\
& \Lambda_{-}(z, t, S, X)=S\left|\varepsilon_{-}(z, t)\right|^{2}+2 X\left|\varepsilon_{+}(z, t)\right|^{2}
\end{aligned}
$$

The quantity $S$ corresponds to the self-phase, SP, modulation term while the quantity $X$ is for the cross-phase, $\mathrm{CP}$, modulation; moreover the term $\Gamma_{a}$ corresponds to the average NL term while the term $\Gamma_{p \Delta}$ is associated with the $p$ th Fourier component of the NL term of the dielectric susceptibility $\Delta \chi^{(3)} u_{p}$.

The matrix $\overline{\mathcal{N}_{c}}$ affecting $\bar{\varepsilon}^{*}(z, t)$ is:

$$
\overline{\mathcal{N}_{c}}=\Gamma_{m}\left(\begin{array}{cc}
\varepsilon_{-}(z, t)^{2} & 0 \\
0 & -\varepsilon_{+}(z, t)^{2}
\end{array}\right)
$$

The coefficient $\Gamma_{m}$ is also related to the $3^{\text {rd }}$ order dielectric susceptibility. It contributes to the wave mixing process. If the polarization is stationary according to our assumption, one can show that in our geometry:

$$
\mathrm{S}=\mathrm{X}=1 ; \Gamma_{a}=\Gamma_{m} \propto 1+4 \pi \bar{\chi}^{(3)}
$$

The loss (or gain) has been taken into account in the above theory by introducing an imaginary part in the dielectric constants. Generally in the x-ray domain, materials are absorbing; nevertheless it has been reported stimulated emission in solid (silicon and magnesium oxide) under X-FEL excitation (Beye et al., 2013; Yoneda et al., 2015; Jonnard et al., 2016), so that the case of medium with gain (lasing medium) needs to be considered too. Let us mention that X-UV lasing in 1D-PC with pumping by X-FEL, forming a so-called distributed feedback laser, has been recently examined (André et al., 2014).

\section{Low-intensity regime: transient response}

In this section we assume that the intensity of the X-FEL radiation is low enough so that no NL behaviour occurs: it is the low-intensity regime, LIR. We will see that, even if the response of the 1D-PC to the pulse is then purely linear, notable effects occur when the time width of the pulse is very short. First we will consider the so-called impulse and indicial response of the 1D-PC in terms of reflection and transmission; the impulse response corresponds to an incident Dirac- $\delta$ pulse while the indicial response corresponds to an incident step-like Heaviside- $\Theta$ signal in terms of time dependence. The 1D-PC is assumed to work at the Bragg resonance.

Under LIR, the system of PDEs, Eq.(7), is linear and can be reduced by means of the following characteristic coordinates $v, w$ :

$$
v=\frac{1}{2}(c t \sin \theta-z)
$$




$$
w=\frac{1}{2}(c t \sin \theta+z)
$$

and reduced field amplitudes defined by:

$$
\begin{aligned}
& \varepsilon_{+}(z, t)=\exp (-i \alpha c t \sin \theta) \tilde{f}(v, w) \\
& \varepsilon_{-}(z, t)=\exp (-i \alpha c t \sin \theta) \tilde{b}(v, w)
\end{aligned}
$$

to the following hyperbolic second order PDE

$$
\mathcal{L}_{(v, w)}[\tilde{f}(v, w) ; \tilde{b}(v, w)]=0
$$

where $\mathcal{L}_{(v, w)}$ is the differential operator defined by:

$$
\mathcal{L}_{(v, w)}=\left(\frac{\partial^{2}}{\partial v \partial w}+\frac{\pi^{2}}{\Lambda^{2}}\right)
$$

with $\Lambda^{2}$, the quantity related to the coupling constants $K^{+}, K^{-}$by:

$$
\Lambda^{2}=-\frac{\pi^{2}}{K^{+} K^{-}}
$$

The quantity $\Lambda$ is the extinction length of the dynamical theory of diffraction. For given boundary conditions, the PDE given by Eq.(14) can be solved by implementing Riemann's method (Courant \& Hilbert, 1965). This method requires an integration contour in the characteristic coordinate plane shown in Figure 2.

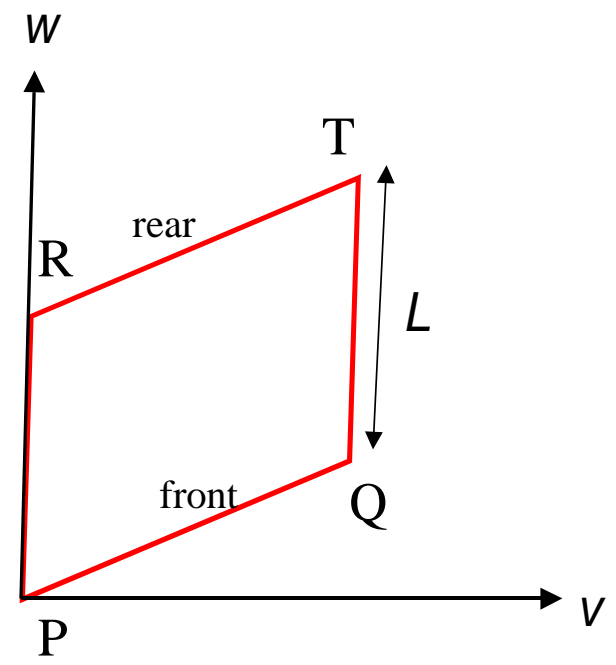

Figure 2: $1 D$ - $P C$ geometry in the characteristic coordinate reference frame $(v, w)$; the front surface is given by $w=v$ (line $P Q$ ) while the rear surface is given by $w=v+L$ (line RT).

Application of Riemann's method leads to write the backward propagating (reflected) field as follows:

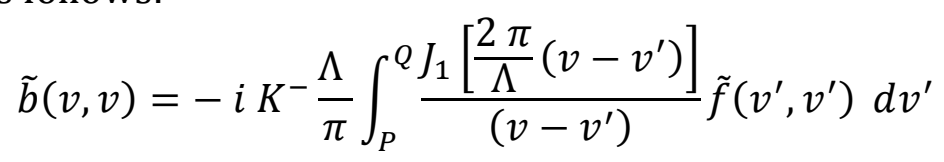

where $\tilde{f}\left(v^{\prime}, v^{\prime}\right)$ corresponds to the incoming (forward propagating) wave at the front surface $(\mathrm{z}=0) . J_{1}$ is the Bessel function of the first kind. From Eq.(17) one can deduce the 
impulse in terms of reflection coefficient. The impulse incident reduced field amplitude $\tilde{f}(v, v)_{\delta}$ can be written as:

$$
\tilde{f}(v, v)_{\delta}=\frac{\exp (+i 2 \alpha v)}{\sqrt{2 \pi}} \frac{\sin \theta}{2} \delta\left[\frac{x \cos \theta \sin \theta}{2}-v\right]
$$

where $\delta$ stands for the Dirac peak. Inserting Eq.(18) in Eq.(17) and performing the integration gives for the reduced diffracted field $\tilde{b}(v, v)_{\delta}$ under the incidence of a Dirac pulse:

$$
\tilde{b}(v, v)_{\delta}=-i \sin \theta K^{-} \frac{\Lambda}{\pi} \frac{\exp (+i a x \cos \theta \sin \theta)}{\sqrt{2 \pi}} \frac{J_{1}\left[\frac{\pi}{\Lambda} \sin \theta(c t-x \cos \theta)\right]}{\sin \theta(c t-x \cos \theta)}
$$

that is, for the diffracted field:

$$
\varepsilon_{-}(z=0, T)_{\delta}=i \sin \theta K^{-} \frac{\Lambda}{\pi \sqrt{2 \pi}} \frac{J_{1}[\zeta(T)]}{\zeta(T)} \Theta(T) ; \zeta(T)=\frac{\pi}{\Lambda} \sin \theta c T
$$

where one has introduced the time delay $T$ measured with respect to the diffracted wave plane:

$$
T=\frac{c t-x \cos \theta}{c}
$$

$\hat{R}_{\delta}(T)=\varepsilon_{-}(z=0, T)_{\delta}$ is the impulse response in terms of reflection and also the temporal Green function $g_{R}(T)$ for reflection. For time coherent radiation with timedependent causal distribution $\Xi$ (normalized to unity), the indicial response $\hat{R}_{\Theta}(t)$ in terms of reflection coefficient is given by:

$$
\hat{R}_{\Theta}(t)=\int_{-\infty}^{+\infty}\left|g_{R}(T)\right|^{2} \Xi(t-T) d T=\int_{0}^{t}\left|g_{R}(T)\right|^{2} \Xi(t-T) d T
$$

Eq.(22) allows one to draw a "universal" curve in terms of peak reflectance for the indicial response $\hat{R}_{\Theta}(t)$ versus the reduced time $\bar{t}=t \frac{\pi \sin \theta c}{\Lambda}$, see Figure 3. It appears that the indicial response for reflection is conditioned by the extinction length $\Lambda$ and that it presents a transient period whose duration is given by a characteristic transient time $t_{c}$ approximately equal to 2 units of reduced time.

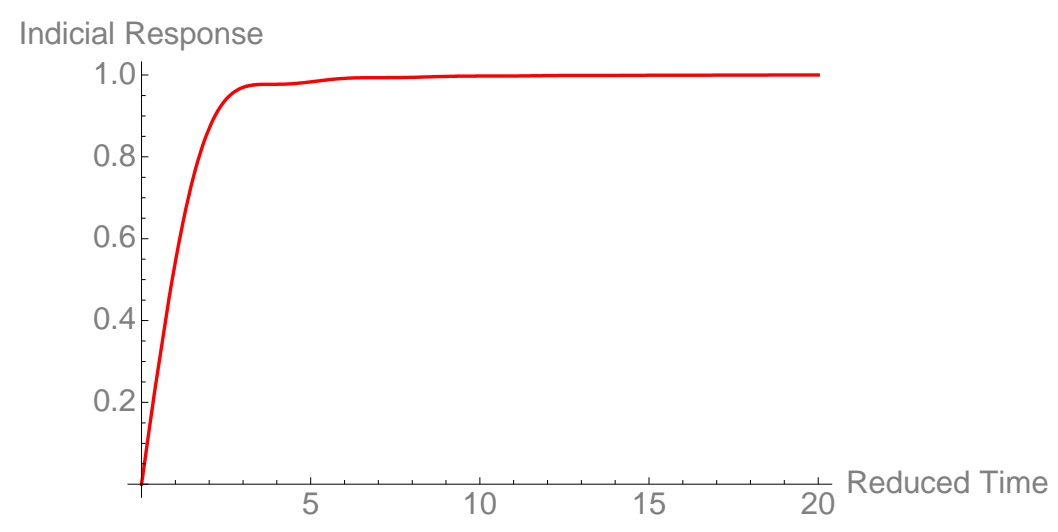

Figure 3: Indicial response in terms of peak reflectance versus reduced time for a $1 D-P C$ at the Bragg resonance (Bragg angle). The response is normalized to unity. 
Following a similar calculation it is possible to show that forward propagating transmitted field $\tilde{f}(v, w)]_{T}$ is given by:

$$
\tilde{f}(v, w)]_{T} \approx-\frac{2 \pi^{2} L}{\Lambda^{2}} \int_{P}^{Q} \tilde{f}\left(v^{\prime}, v^{\prime}\right) \frac{J_{1}\left[\frac{2 \pi}{\Lambda} \sqrt{\left(v+L-v^{\prime}\right)\left(v-v^{\prime}\right)}\right]}{\frac{2 \pi}{\Lambda} \sqrt{\left(v+L-v^{\prime}\right)\left(v-v^{\prime}\right)}} d v^{\prime}
$$

To determine the impulse response in terms of transmission, we insert in Eq.(22) the expression of the incident pulse given by Eq.(18) as for the reflection case and we perform the integration as for the reflection geometry. It results that the impulse response in terms of transmission $\widehat{T}_{\delta}(t)$ is $\varepsilon_{+}(z=L, T)_{\delta}$ :

$$
\varepsilon_{+}(z=L, T)_{\delta}=\frac{\pi^{2} L}{\sin \theta \Lambda^{2}} \frac{J_{1}[\xi(T)]}{\xi(T)} \Theta(T) ; \xi(T)=\frac{\pi}{\Lambda} \sqrt{c T\left(\frac{2 L}{\sin \theta}+\frac{c T}{\sin \theta^{2}}\right)}
$$

For time coherent radiation with time-dependent causal distribution $\Xi$ the indicial response $\widehat{T}_{\Theta}(t)$ in terms of transmission coefficient is given from the temporal Green function for transmission $g_{T}=\widehat{T}_{\delta}$ :

$$
\widehat{T}_{\Theta}(t)=\int_{-\infty}^{+\infty}\left|g_{T}(T)\right|^{2} \Xi(t-T) d T=\int_{0}^{t}\left|g_{T}(T)\right|^{2} \Xi(t-T) d T
$$

The indicial response in terms of transmission is conditioned both by the extinction length $\Lambda$ and by the thickness $L$ of the 1D-PC.

From the indicial responses of the $1 \mathrm{D}-\mathrm{PC}$ it is possible to calculate the timedependent reflection and transmission of a short pulse. Let $I(t)$ be the temporal envelope shape of any incident pulse and $O(t)$ the envelope of the corresponding reflected or transmitted pulse. Then the Laplace transform $O(s)$ of $O(t)$ is related to the Laplace transform $I(s)$ of $I(t)$ by means of the convolution theorem:

$$
O(s)=\widehat{\mathrm{Z}}_{\delta}(s) I(s)
$$

$\widehat{\mathrm{Z}}_{\delta}(s)$ being the transfer function that is the Laplace transform of the impulse response $\widehat{\mathrm{Z}}_{\delta}(t)\left(\equiv \hat{R}_{\delta}(t)\right.$ or $\left.\widehat{T}_{\delta}(t)\right) . \hat{\mathrm{Z}}_{\delta}(t)$ can be determined from $\hat{\mathrm{Z}}_{\Theta}(t)$ by Strejc's method (de Larminat, 2007). By performing an inverse Laplace transform of Eq.(26), one determines the temporal response (André \& Jonnard, 2015).

We now illustrate the previous theory in the case of a MIM consisting in a stack of $\mathrm{N}=7 \mathrm{Ti} / \mathrm{Si}$ bilayers; the period $d$ is equal to $70 \mathrm{~nm}$ and the $\gamma$ ratio is equal to 0.5 . The photon energy of the incident XFEL radiation is $20 \mathrm{eV}(\lambda=62 \mathrm{~nm})$ diffracted at $\theta=60^{\circ}$. The choice of the Ti/Si system is not governed by the objective of optimizing the reflectance of the MIM but by the fact that it has been shown, see (Bencivenga et al., 2014), that titanium has a NL behaviour at the photon energy of interest. The temporal profile XFEL pulse is modelled by a sine-squared function, that is the envelope of the incident pulse varies as $\sin ^{2}\left(\pi \frac{t}{\tau}\right)$ in the time interval $[0, \tau]$ and 0 outside.

The NL dielectric constant (square of the refractive index) of titanium is assumed to be given by Drude's formula modified to account for the intensity dependence (Bencivenga et al., 2014): 


$$
\begin{gathered}
\epsilon_{N L T i}=1-\frac{\omega_{p 0}^{2}(1+A \overline{\mathrm{E}})}{\omega^{2}+i \omega \bar{\gamma}} \approx \epsilon_{L T i}+\Delta \epsilon_{T i} \overline{\mathrm{E}} \\
\epsilon_{L T i}=1-\frac{\omega_{p 0}{ }^{2}}{\omega^{2}+i \omega \bar{\gamma}} \approx 1-\frac{\omega_{p 0}^{2}}{\omega^{2}}+i \bar{\gamma} \frac{\omega_{p 0}^{2}}{\omega^{3}} ; \Delta \epsilon_{T i}=-A \frac{\omega_{p 0}{ }^{2}}{\omega^{2}}
\end{gathered}
$$

$\omega_{p 0}$ is the plasma frequency at zero energy density $(\overline{\mathrm{E}}=0)$ equal to $17.7 \mathrm{eV}, \mathrm{A}$ being an empirical constant equal to $7.510^{12} \mathrm{~m}^{3} / \mathrm{J}$. Following (Bencivenga et al., 2014) the damping term $\bar{\gamma}$ is taken to be $0.1 \omega_{p 0}$ as for aluminum (Ujihara, 1972) since a reference value is not available in the literature for Ti. Numerically at $20 \mathrm{eV}$, one has:

$$
\epsilon_{L T i}=0.217+i 0.069=(0.474+i 0.087)^{2} ; \Delta \epsilon_{T i}=-5.8710^{12}
$$

For silicon, from (Palik, 1985), at $20 \mathrm{eV}$ :

$$
\epsilon_{L S i}=(0.567+i 0.083)^{2}
$$

Figure 4 displays the indicial response in terms of reflectance for the Ti/Si MIM under consideration, for different glancing angles close to the Bragg angle $\left(\theta=60^{\circ}\right)$ obtained by solving Eq.(7), (André \& Jonnard, 2015). It appears that the transient time is around 6.5 fs to reach $90 \%$ of the stationary indicial response. The temporal response in terms of peak reflectance (that is for resonance at Bragg angle $\theta=60^{\circ}$ ) to a single pulse for several time widths $\tau(10,50,100 \mathrm{fs})$ is shown in Figure 5. It can be seen that for the shortest incident pulses, the reflectance does not have enough time to reach its steadystate value. For the longest pulses the response duration is quite symmetrical and lasts about the time of the pulse. For the shortest pulse, the response is asymmetrical and lasts at least twice the time of the pulse.

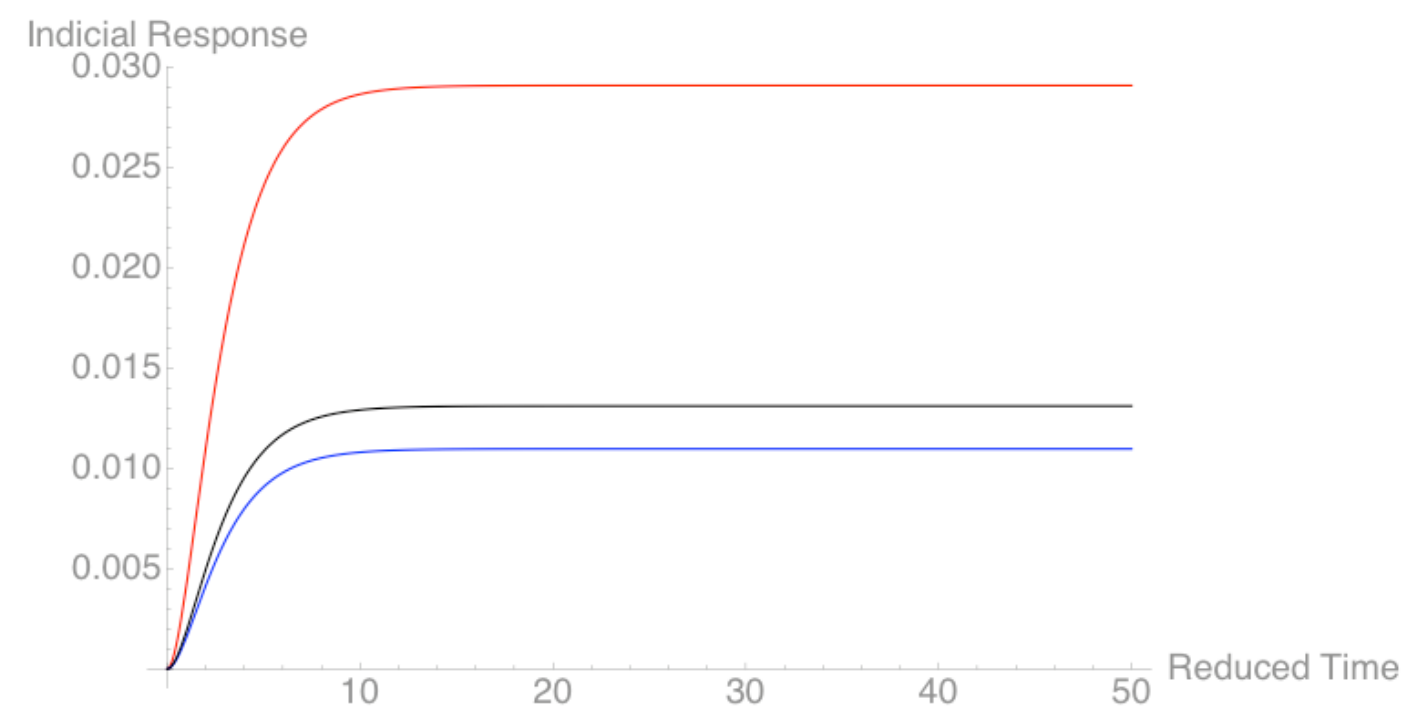

Figure 4: Indicial response of the Ti/Si MIM versus time in terms of peak reflectance for different glancing angles $\theta$ : red line, $\theta=60^{\circ}$ (Bragg angle); black line, $\theta=65^{\circ}$; blue line $\theta=55^{\circ}$. 


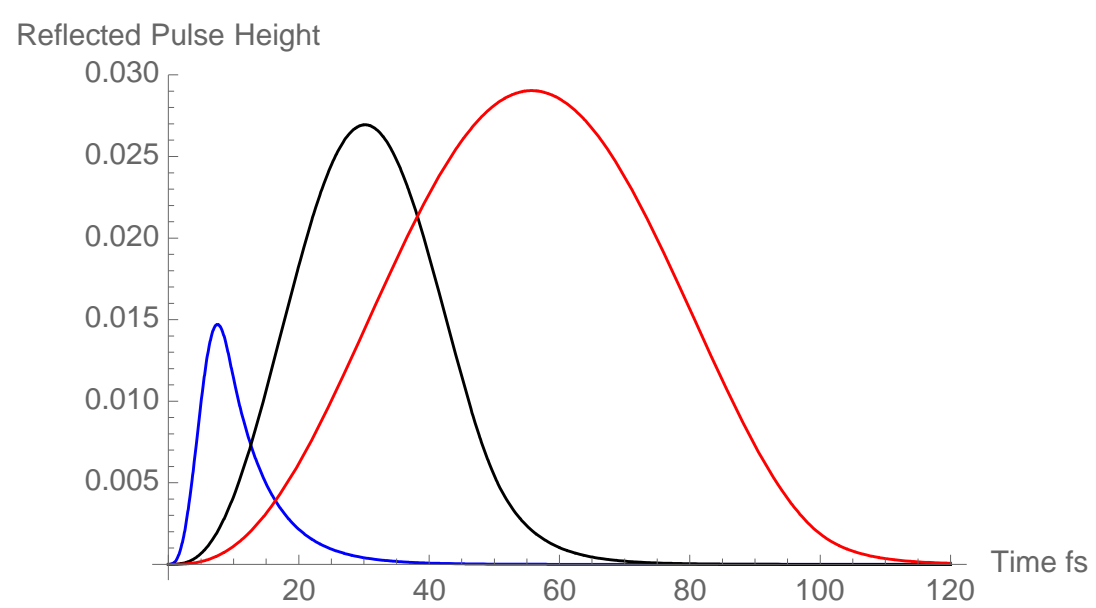

Figure 5: Reflected pulse by the Ti/Si MIM at Bragg resonance versus time for several time

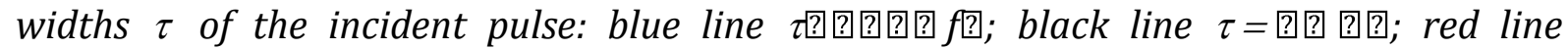

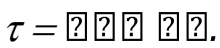

\section{High-intensity regime HIR: solitons and other non-linear effects}

In this part, our purpose is to highlight some NL effects occurring in the highintensity regime where Kerr non-linearity takes place. If one starts from the general CW equation Eq.(7) to deal with this regime, one has to face with a lot of mathematical problems very difficult to handle, leaving one with only numerical techniques generally computationally intensive and that do not provide the necessary insight into the physics. So we choose to simplify the problem by keeping in the Eq.(7) only the terms which describe the basic phenomena. In this spirit we restrict our study to the following cases:

- loss and/or gain in the medium are discarded;

- the 1D-PC is tuned at the $p$ th Bragg resonance;

- wave mixing that is the effect of the matrix $\overline{\mathcal{N}_{c}}$ in Eq.(7) is neglected.

\subsection{NL stationary case}

From the study of the time-dependent linear case examined in section 3, one can expect for pulses with time width very large with respect to the characteristic time associated to the extinction length, that the propagation of the pulse in NL media can be satisfactorily described by the time independent (stationary) form of the NL PDEs. It can be shown that in these conditions, the system of hyperbolic PDEs deduced from Eq.(7) with the above-mentioned assumptions has two conserved quantities depending on $\varepsilon_{+}(z)$ and $\varepsilon_{-}(z)$ :

- the intensity flow: $\left|\varepsilon_{+}(z)\right|^{2}-\left|\varepsilon_{-}(z)\right|^{2}=I_{\text {in }}-I_{\text {ref }}=I_{\text {out }} \equiv T$ (see Appendix);

- the real-valued Hamiltonian (non-explicited):

and since the degree of freedom (equal to 2) of the PDE system is equal to the number of conserved quantities, by virtue of the Liouville-Arnould theorem, this PDE system is exactly integrable. With the "standard" boundary conditions,

$$
\left|\varepsilon_{+}(0)\right|^{2}=I_{\text {in }},\left|\varepsilon_{-}(0)\right|^{2}=I_{\text {ref }},\left|\varepsilon_{+}(L)\right|^{2}=I_{\text {out }},\left|\varepsilon_{-}(L)\right|^{2}=0
$$

the integration yields relationships between the transmittance (reflectance) and the input intensity $I_{\text {in }}$ in terms of Jacobi elliptic functions (see Appendix). From the expression of the transmittance deduced from Eq.(A.7), it appears that the 1D-PC can present a bi-stability. This is illustrated by the figure 6 obtained from Eq.(A.12). It shows a typical $S$-shape response at Bragg resonance in terms of normalized transmittance 
versus $I_{\text {in }}$ for the value of $\bar{\kappa} L$ equal to 2; the negative slope region (between 4.75 and $4.85)$ is unstable. The Bragg reflectivity associated with the linear component of the dielectric constant is erased until a transparent state (reflectivity close to zero) is reached at a point $\mathrm{T}$ as shown in Figure 6. For low value of $\bar{\kappa} L$, bistability cannot be achieved by lack of feedback but for larger values of $\bar{\kappa} L$, multiple-switching phenomena appear (Winful et al., 1979). NL 1D-PC can give rise according, to the conditions, to multi-stabilities as well as optical limiting (see section 4.2 .2 of the paper) and even may undergo chaotic behavior.

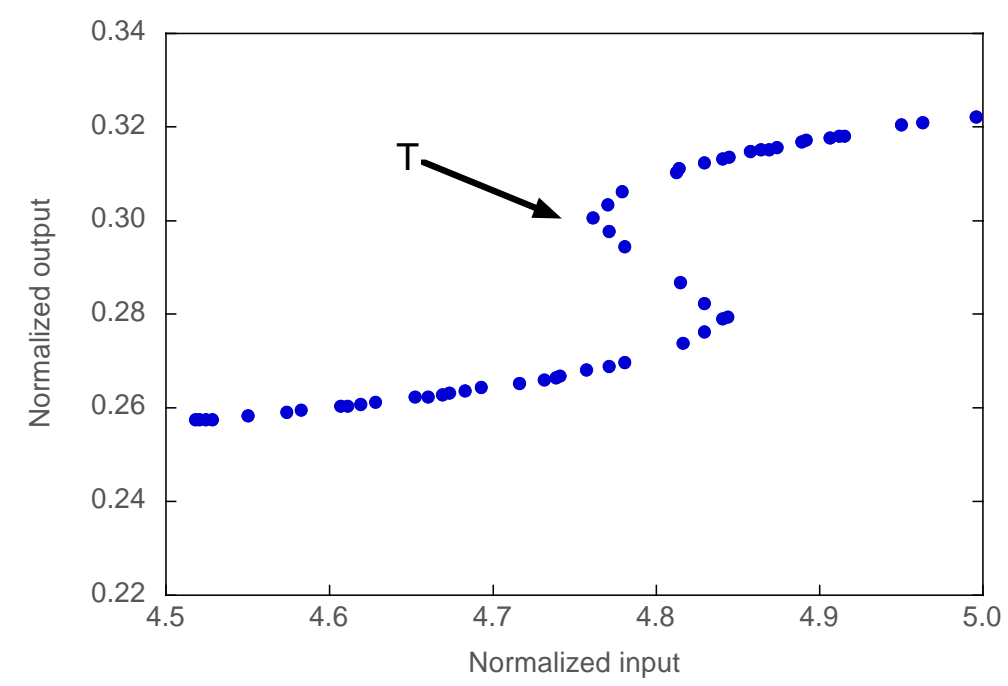

Figure 6: Transmittance versus normalized input intensity exhibiting bi-stability of the NL $1 D$-PC; a quasi-transparent state appears at the point $T$.

\subsection{NL time-dependent case 4.2.1 Solitons}

The physics of the 1D-PC in the linear case is governed by the occurrence of two branches of the dispersion curve in the frequency domain, experiencing anti-crossing owing to the wave coupling. In the contra-propagating case relevant for our problem, the anti-crossing gives rise to a forbidden energy gap. In the LIR considered in the previous section, the Bragg frequency $\omega_{c}$ lies within the band gap where running waves are forbidden. At HIR we are dealing with in this part, the dielectric constant changes proportionally to the electric field intensity and the branches shift down/up in energy (a kind of blue/red shift of the band gap according to positive/negative NL) so that the frequency $\omega_{c}$ no longer falls within the forbidden gap but shifts towards a region with allowed traveling waves. This mechanism is sketched in Figure 7. The gap soliton and the optical limiting and switching examined hereafter are related to this mechanism. 
(a) LIR

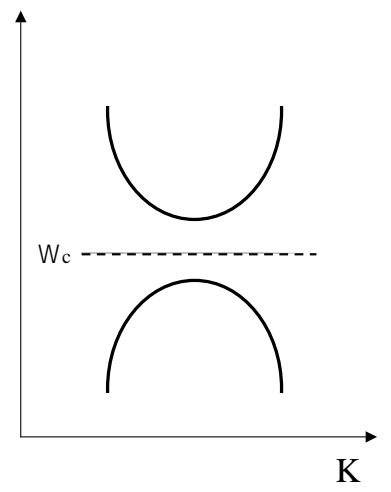

(b) HIR

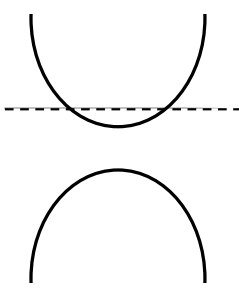

(c) HIR

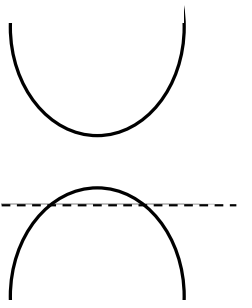

Figure 7: Dispersion curves in the frequency ( $\omega)$ / Bloch wavenumber (K) domain: (a) LIR; (b) HIR and positive NL with a shift-down in frequency; (c) HIR and negative NL with a shift-up in frequency.

The dynamics of the system is then governed by of the system of NL PDEs Eq.(7). A remarkable property of this system is that the entire family of localized solutions can be built analytically forming the "soliton-like" solutions. The adequacy of the term "soliton" is discussed in many specialized works, see for instance (Wazwaz, 2009); here we merely use the term soliton to mean localized solutions not in the strict mathematical sense of integrability. We first consider the case where SP is neglected $(S=0)$. Although one could imagine cases for which $X=0$, it seems that there are no realistic geometries in which $S=0$ and $X=0$ so that the model in these conditions is not relevant directly in our problem. Nevertheless the latter case paves the way to a general solution as we will see hereafter. It has been recognized that the system given by Eq.(6) with $S=0$ and $X \neq 0$ reduces to the massive Thirring model MTM which is integrable and exhibits soliton-like solutions (Martun de Sterke \& Sipe, 1994). The more general solution when the SM term is not neglected (generally $\mathrm{X}=\mathrm{S}$ ) can be built from the soliton-like solutions of the MTM (Orfanidis \& Wang, 1975).

We briefly present the mathematical way to the more general (in the sense $S \neq$ $0, X \neq 0$ and $\operatorname{Im}(\varepsilon) \neq 0$ but wave mixing terms remain null) soliton-like solution of Eq.(6). First the column vector $\bar{\varepsilon}(z, t)$ without SM can be regarded as a Dirac spinor $\psi$ and Eq.(6) is formally the same than the MTM equation:

$$
i \gamma^{\mu} \partial_{\mu} \psi=m \psi-g J_{\mu} \psi ; J^{\mu}=\bar{\psi} \gamma^{\mu} \psi
$$

$\gamma^{\mu}$ being the gamma matrices formed from the Pauli matrices $\sigma_{\mu}$. The MTM equation can be reduced to the Sine-Gordon one which is integrable; finally the soliton-like solutions of the MTM are (Aceves \& Wabnitz, 1989):

$$
\psi=\left(\begin{array}{l}
\psi_{1} \\
\psi_{2}
\end{array}\right)
$$

with

$$
\left.\psi_{1}= \pm\left( \pm \frac{\bar{\kappa}}{2 \Gamma_{a}}\right)^{1 / 2} \frac{1}{\Delta} \sin Q \exp [ \pm i \bar{\kappa} \zeta \cos Q] \operatorname{sech}[\bar{\kappa} \xi \sin Q \mp i Q / 2)\right]
$$




$$
\begin{gathered}
\left.\psi_{2}=-\left( \pm \frac{\bar{\kappa}}{2 \Gamma_{a}}\right)^{1 / 2} \Delta \sin Q \exp [ \pm i \bar{\kappa} \zeta \cos Q] \operatorname{sech}[\bar{\kappa} \xi \sin Q \pm i Q / 2)\right] \\
\zeta=\frac{v z-t}{\sqrt{1-v^{2}}} \\
\xi=\frac{z-v t}{\sqrt{1-v^{2}}}
\end{gathered}
$$

The signs are determined by the relative sign of the linear and NL coupling coefficients. Eqs.(30) represent a two parameter family of solutions of the system Eq.(7) with $\Delta$ and $Q$ as two free parameters which are respectively obtained from the amplitude and phase of the eigenvalue of the scattering problem connected to MTM; $v$ is a dimensionless quantity $(|v|<1)$ given by:

$$
v=\frac{1-\Delta^{4}}{1+\Delta^{4}}
$$

that determines the velocity of the soliton. The parameter $Q(0<Q<\pi)$ gives the location of the soliton in the band-gap and determines its width: $Q=\frac{\pi}{2}$ corresponds to a soliton with center frequency in the middle of the gap reducing to the slow Bragg soliton of (Leung, 1985); the limit $Q \rightarrow 0$ corresponds to soliton-like solutions at the top of the gap reducing to the NL Schrödinger one-soliton solution of the NL PDE (Sipe \& Winful, 1988) whereas the limit $Q \rightarrow \pi$ correspond to plane wave solutions. The generalized (i.e. the SM is not neglected) soliton-like solution $\bar{\varepsilon}(z, t)$ is built from the soliton solutions $\psi$ of the MTM given by Eqs(18):

$$
\bar{\varepsilon}=\mathcal{A} \psi \exp (i \vartheta(\xi))
$$

with

$$
\begin{aligned}
\mathcal{A} & =\frac{1}{\sqrt{1+R_{+}+R_{-}}} \\
R_{ \pm} & =\frac{S}{4 \mathrm{X}} \frac{(1 \pm v)^{2}}{1-v^{2}} \\
\exp (i \vartheta(\xi)) & =\left(-\frac{e^{2 \bar{\kappa} \xi \sin Q}+e^{\mp i Q}}{e^{2 \bar{\kappa} \xi \sin Q}+e^{ \pm i Q}}\right)^{S} \\
s & =\frac{R_{+}-R_{-}}{\mathcal{A}^{2}}
\end{aligned}
$$

The solitons given by Eq.(32) are called self-transparency solitons. We note that the NL interaction effects appear in Eq.(32) mainly through the quantities $R_{ \pm}$via the ratio $\frac{\mathrm{S}}{4 \mathrm{X}}$. Figure 8 shows the time evolution of the shape of a slow Bragg soliton formed in the 1DPC. 


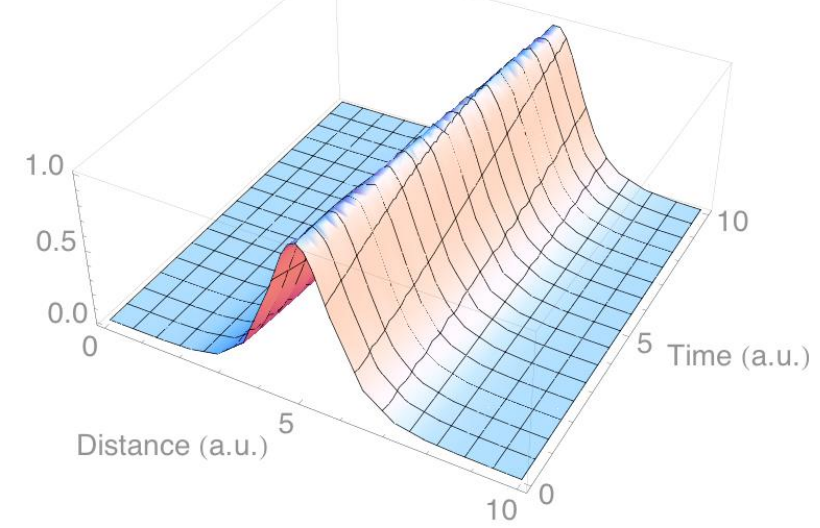

Figure 8: Time evolution of the shape of a slow Bragg soliton in arbitrary units, formed in the $1 D-P C$ in the NL regime.

These soliton-like solutions can be pictured by an effective particle (Martijn de Sterke \& Sipe, 1989) with a charge $\mathbf{q}$ and a momentum $\mathbf{p}$ :

$$
\begin{gathered}
\mathbf{q}=\int_{-\infty}^{+\infty}\left(\varepsilon^{*}{ }_{+} \varepsilon_{+}+\varepsilon^{*} \varepsilon_{-}\right) d z \\
\mathbf{p}=-i \int_{-\infty}^{+\infty}\left(\varepsilon^{*}{ }_{+} \partial_{z} \varepsilon_{+}+\varepsilon^{*}{ }_{-} \partial_{z} \varepsilon_{-}\right) d z
\end{gathered}
$$

Using Eq.(7), it comes:

$$
\partial_{z} \mathbf{q}=2 \operatorname{Im}(K) \mathbf{q}
$$

and

$$
\partial_{t} \mathbf{p}=2 \operatorname{Im}(\mathrm{K}) \mathbf{p}
$$

It appears that in absence of loss or gain, both momentum and charge will be conserved quantities, otherwise they vary exponentially with time. From these equations it can be shown that the important parameter $Q$ satisfies:

$$
\partial_{t} Q=2 \operatorname{Im}(K) Q
$$

From Eq.(37) it follows that the width of the soliton which is determined by Q, depends exponentially on time while its velocity $v$ is still undetermined. Detailed analysis concerning the behaviour of gap solitons in 1D-PC made up with NL media with loss and gain can be found in (Martijn de Sterke \& Sipe, 1991).

As mentioned previously, the velocity $v$ can be regarded as a free parameter; in other words by choosing the conditions appropriately, it should be possible to get very slow solitons, and even stationary solitons, up to solitons moving at about the speed of light. Low velocity $(v \approx 0)$ means that energy will be transported very slowly at the manner of the self-induced transparency, SIT, observed in resonant pulse propagation in atoms (McCall \& Hahn, 1967); one can then speak about Bragg SIT. Let us mention the possible existence of multi-soliton-like solutions of the time-dependent NL problem.

We now turn to the study and the conditions of a possible soliton under X-FEL pulse irradiation. First let us outline that the problem of finding the right features of the incident pulse and the appropriate conditions to give rise to a given soliton is far from trivial. For a finite length MIM with $\mathcal{N}$ unit cells (bilayers), Martijn de Sterke and Sipe 
(Martijn de Sterke \& Sipe, 1989) have given the following criteria to satisfy for the occurrence of a gap soliton:

$$
\mathcal{N}^{2} \Delta n_{L} \Delta n_{N L} \geq 1
$$

where $\Delta n_{L}$ is the magnitude of the varying term of the refractive index and $\Delta n_{N L}$ the maximum NL change in the refractive index. Let us also emphasize that the problem of the soliton stability is a hard task (Hwang et al., 2011) out of the domain of this paper.

\subsubsection{Optical switching}

The dynamics of NL 1D-PC offers the possibility of a large variety of mechanisms very attractive for practical applications: optical limiting and switching, pulse generation or reshaping, ... In the following we consider the optical limiting and switching OLS, in further details because of its potential interest in x-ray technology. OLS results from a dynamical change of the NL 1D-PC response to an incident field intensity. To understand the principle of the OLS mechanism, it is convenient to consider the frequency domain. In this one, the width of the forbidden gap (Bragg domain), GW, is proportional to the difference of dielectric constants $\Delta \epsilon_{\boldsymbol{a b}}$ between material $\mathbf{a}$ and $\mathbf{b}$. Let us assume that one the material, say a, presents a NL Kerr-like behavior with positive coefficient, i.e. its dielectric constant increases with the incident field intensity $\overline{\mathrm{E}}$ while the other material as a linear behaviour. Then as $\bar{E}$ increases, GW increases too, so that a dynamical widening of the gap occurs. If the carrier frequency of the incident radiation is tuned at the band edge, then a larger domain of frequency of the incident pulse will fall inside the forbidden gap until forbidding the complete propagation of the pulse. This mechanism described in (Scalora et al., 1994) forms the basis of an intensity-driven OLS.

For a NL behavior with negative coefficient, switching can occur from the linear material in the pump/probe scheme sketched in Figure 9: a strong pump pulse of carrier frequency $\omega_{p p}$ far below the gap and of intensity $\overline{\mathrm{E}}_{p p}$ inducing the non-linearity and a probe pulse of carrier frequency $\omega_{p r}$ inside the gap but near the band edge and of intensity $\overline{\mathrm{E}}_{p r} \ll \overline{\mathrm{E}}_{p p}$ are incident on the 1D-PC. Initially (i.e. without pumping) the probe pulse is not transmitted. For negative coefficient, under pump beam irradiation, the bandwidth GW decreases and over a certain value of $\overline{\mathrm{E}}_{p p}$, the probe frequency $\omega_{p r}$ may exit the gap leading to a transmission of the probe beam. This process, which can be encountered with the negative NL coefficient of Ti in our example, may form the basis of an x-ray switch.

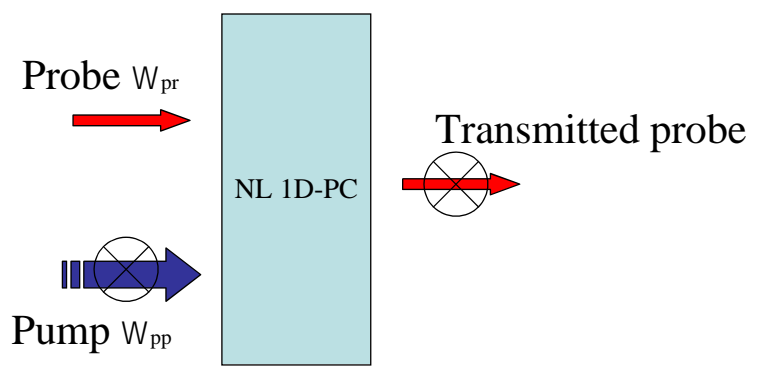

Figure 9: Scheme for an optical switching with a NL 1D-PC with negative coefficient. The $N L$ Kerr effect is induced by the pump beam and can allow the transmission of the probe beam. In absence of pump, there is no transmitted probe; this case is illustrated by the cross on the arrows.

\section{Conclusion}


Ultra-short or ultra-intense x-ray pulses delivered by the X-FEL propagating within 1D-PC opens the way to the observation of a lot of phenomena never encountered in the x-ray regime. In fact none of the phenomena considered in this paper have been evidenced until now and their observation is technically challenging. Considering the LIR, the measurement of the intensity of a reflected single pulse remains tricky. Nevertheless techniques as those implemented at the TIMEX end-station in FERMI X-FEL facility to measure the reflectivity (Bencivenga et al., 2014) can be envisaged to test our model.

When NL is incorporated into the 1D-PC at HIR, it has been shown that it becomes possible to control dynamically the propagation of ultra-intense x-ray pulse. NL effects are generally studies both experimentally and theoretically for lossless media; in the x-ray domain and in particular for the soft x-ray range, the consequence of absorption on the NL effects are difficult to forecast in the CW theory framework without intensive numerical computation. For this purpose, calculation implementing transfer matrix method extended to Kerr NL in a way similar to (Li et al., 2015) could be envisaged. From the experimental point of view, to test NL effects, techniques borrowed from the optical domain involving pulse compression with cross-phase modulation should be implemented (Martijn de Sterke, 1992). 


\section{Appendix}

With the assumptions mentioned in the main text, the stationary version of the PDE system given by Eq.(7) becomes at the $p t h$ Bragg resonance:

$$
i \partial_{z} \varepsilon_{ \pm}(z)=\mp \bar{\alpha}\left[\left(\left|\varepsilon_{ \pm}(z)\right|^{2}+2\left|\varepsilon_{\mp}(z)\right|^{2}\right)\right] \varepsilon_{ \pm}(z)+\bar{\kappa} \varepsilon_{\mp}(z)
$$

with

$$
\bar{\alpha}=\frac{k^{2}}{\kappa} 2 \pi \bar{\chi}^{(3)} ; \bar{\kappa}=\frac{k^{2}}{\kappa} 2 \pi \Delta \chi^{(1)} u_{p}
$$

The quantity $T=\left|\varepsilon_{+}(z)\right|^{2}-\left|\varepsilon_{-}(z)\right|^{2}$ corresponding to the transmitted flux is a conserved quantity (i.e. $z$ independent). Indeed setting $\varepsilon_{ \pm}(z)=\left|\varepsilon_{ \pm}(z)\right| \exp \left( \pm \phi_{ \pm}(z)\right)$ and subtituting into Eq.(A.1) gives after separation of the real and imaginary parts:

$$
\partial_{z}\left|\varepsilon_{ \pm}(z)\right|=\bar{\kappa}\left|\varepsilon_{\mp}(z)\right| \sin \psi
$$

and

$$
\left|\varepsilon_{ \pm}(z)\right| \partial_{z} \phi_{ \pm}=\bar{\kappa}\left|\varepsilon_{\mp}(z)\right| \cos \psi+\bar{\alpha}\left[\left(\left|\varepsilon_{ \pm}(z)\right|^{2}+2\left|\varepsilon_{\mp}(z)\right|^{2}\right)\right]\left|\varepsilon_{ \pm}(z)\right|
$$

where $\psi(z)=\phi_{+}(z)-\phi_{-}(z)$ at the Bragg resonance. Elimination of $\psi(z)$ from Eq.(A.3) and Eq.(A.4) gives the constant of propagation $T$. Introducing $T$ in (A.1) leads to:

$$
\left(\partial_{z}\left|\varepsilon_{+}(z)\right|^{2}\right)^{2}=4\left|\varepsilon_{+}(z)\right|^{2}\left(\left|\varepsilon_{+}(z)\right|^{2}-T\right)\left(\bar{\kappa}^{2}-9 \bar{\alpha}^{2}\left|\varepsilon_{+}(z)\right|^{2}\left(\left|\varepsilon_{+}(z)\right|^{2}-T\right)\right)
$$

Using the following new quantities:

$$
\rho=\frac{\left|\varepsilon_{+}(z)\right|^{2}}{\left|\varepsilon_{-}(z)\right|^{2}}, Z=\frac{2 z}{L}, N=\frac{2}{3 \bar{\alpha}}, \quad I_{\text {out }}=\frac{T}{N}
$$

Eq.(A.1) can be rewritten:

$$
\frac{d \rho}{d Z}= \pm \sqrt{\rho\left(\rho-I_{\text {out }}\right)\left[(\bar{\kappa} L)^{2}-4 \rho\left(\rho-I_{\text {out }}\right)\right]}
$$

that is taking into account the "standard" boundary conditions Eqs.(27), Eq.(A.6) gives:

$$
\int_{0}^{\infty} \frac{d \rho}{\sqrt{\rho\left(\rho-I_{\text {out }}\right)\left[(\bar{\kappa} L)^{2}-4 \rho\left(\rho-I_{\text {out }}\right)\right]}}=2
$$

where $\rho_{0}^{ \pm}$are the two roots of the binomial equation $(\bar{\kappa} L)^{2}-4 \rho\left(\rho-I_{\text {out }}\right)=0$.

Evaluation of the integral on the left side of Eq.(A.2) depends on the relative value of the roots of the quadric polynomial under the root square. In most usual situations, these roots are real and satisfy:

$$
\rho(Z=2) \rightarrow \infty>\rho_{0}^{+}>I_{\text {out }}>0>\rho_{0}^{-}
$$

In this condition, the integral on the right-hand side of Eq. (A.7) can be found by using the method given in (Byrd \& Friedman, 1971). After some algebra:

$$
\rho(Z)=\frac{I_{\text {out }}}{2}(1+n d[u(Z) / m])
$$


$n d[u(Z) / m]$ being the inverse of the delta amplitude of the Jacobian elliptic (cnoidal) function $d n[u(Z) / m]$ ) (Byrd \& Friedman, 1971), where the modulus $m$ is given by:

$$
m=\sqrt{\frac{(\bar{\kappa} L)^{2}}{(\bar{\kappa} L)^{2}+I_{\text {out }}^{2}}}
$$

and

$$
u(Z)=2 \sqrt{(\bar{\kappa} L)^{2}+I_{\text {out }}^{2}}\left(1-\frac{Z}{2}\right)
$$

The quantity $\rho(Z=0)$ corresponds to normalized input intensity $I_{\text {in }}$ and from Eq.(A.9) one deduces the relation between $I_{\text {out }}$ and $I_{\text {in }}$ :

$$
I_{\text {out }}=\frac{2 I_{\text {in }}}{1+n d\left[2 \sqrt{(\bar{\kappa} L)^{2}+I_{\text {out }}^{2}} / m\right]}
$$

In the NL regime, one recovers the well-known expression for the transmission at Bragg resonance:

$$
I_{\text {out }}=I_{\text {in }} \operatorname{sech}^{2}(\bar{\kappa} L)
$$




\section{REFERENCES}

Aceves, A. B. \& Wabnitz, S. (1989). Phys. Lett. A. 141, 37-42.

André, J.-M. \& Jonnard, P. (2015). J. Opt. 17, 85609.

André, J.-M., Le Guen, K. \& Jonnard, P. (2014). Laser Phys. 24, 85001.

Authier, A. (2003). Dynamical Theory of X-Ray Diffraction International Union of Crystallography Monographs on Crystallography 11.

Bencivenga, F., Principi, E., Giangrisostomi, E., Cucini, R., Battistoni, A., D’Amico, F., Di Cicco, A., Di Fonzo, S., Filipponi, A., Gessini, A., Gunnella, R., Marsi, M., Properzi, L., Saito, M. \& Masciovecchio, C. (2014). Sci. Rep. 4, 4952.

Beye, M., Schreck, S., Sorgenfrei, F., Trabant, C., Pontius, N., Schüßler-Langeheine, C., Wurth, W. \& Föhlisch, A. (2013). Nature. 501, 191-194.

Bushuev, V. A. (2008). J. Synchrotron Radiat. 15, 495-505.

Bushuev, V. \& Samoylova, L. (2011). Nucl. Instrum. Methods Phys. Res. Sect. Accel. Spectrometers Detect. Assoc. Equip. 635, S19-S23.

Byrd, P. F. \& Friedman, M. D. (1971). Handbook of Elliptic Integrals for Engineers and Scientists Berlin, Heidelberg: Springer-Verlag.

Chen, W. \& Mills, D. L. (1987). Phys. Rev. Lett. 58, 160-163.

Chukhovskii, F. N. \& Förster, E. (1995). Acta Crystallogr. A. 51, 668-672.

Courant, R. \& Hilbert, D. (1965). Partial Differential Equations Interscience Publishers.

Hwang, G., Akylas, T. R. \& Yang, J. (2011). Phys. D. 240, 1055-1068.

Jonnard, P., André, J.-M., Le Guen, K., Wu, M., Principi, E., Simoncig, A., Gessini, A., Mincigrucci, R. \& Masciovecchio, C. (2016). EUV stimulated emission from MgO pumped by FEL pulses, https://hal.archives-ouvertes.fr/hal-01344717.

Ksenzov, D., Grigorian, S., Hendel, S., Bienert, F., Sacher, M. D., Heinzmann, U. \& Pietsch, U. (2009). Phys. Status Solidi A. 206, 1875-1879.

Ksenzov, D., Grigorian, S. \& Pietsch, U. (2008). J. Synchrotron Radiat. 15, 19-25.

de Larminat, P. (2007). Analysis and Control of Linear Systems London, Newport Beach: ISTE Ltd.

Leung, K. M. (1985). Phys. Rev. B. 32, 5093-5101.

Li, H., Haus, J. W. \& Banerjee, P. P. (2015). J. Opt. Soc. Am. B. 32, 1456-1462.

Martijn de Sterke, C. \& Sipe, J. E. (1989). Phys. Rev. A. 39, 5163-5178.

Martijn de Sterke, C. \& Sipe, J. E. (1991). Phys. Rev. A. 43, 2467-2473.

Martijn de Sterke, C. (1992). Opt. Lett. 17, 914-916.

Martijn de Sterke, C. \& Sipe, J. E. (1994). Progress in Optics, Vol. Volume 33, edited by E. Wolf, pp. 203-260. Elsevier.

McCall, S. L. \& Hahn, E. L. (1967). Phys. Rev. Lett. 18, 908-911.

Mills, D. L. \& Trullinger, S. E. (1987). Phys. Rev. B. 36, 947-952.

New, G. (2014). Introduction to Nonlinear Optics Cambridge: Cambridge University Press.

Orfanidis, S. J. \& Wang, R. (1975). Phys. Lett. B. 57, 281-283.

Palik, E. D. (1985). Handbook of Optical Constants of Solids Boston: Academic Press. Pardo, B., Megademini, T. \& André, J.-M. (1988). Rev. Phys. Appliquée. 23, 1579-1597.

Scalora, M., Dowling, J. P., Bowden, C. M. \& Bloemer, M. J. (1994). Phys. Rev. Lett. 73, 1368-1371.

Shastri, S. D., Zambianchi, P. \& Mills, D. M. (2001). J. Synchrotron Radiat. 8, 1131-1135.

Sipe, J. E. \& Winful, H. G. (1988). Opt. Lett. 13, 132-133.

Ujihara, K. (1972). J. Appl. Phys. 43, 2376-2383.

Wazwaz, A.-M. (2009). Partial Differential Equations and Solitary Waves Theory Dordrecht, Heidelberg, London, New York: Springer. 
Winful, H. G. (1985). Appl. Phys. Lett. 46, 527-529.

Winful, H. G., Marburger, J. H. \& Garmire, E. (1979). Appl. Phys. Lett. 35, 379-381.

Yoneda, H., Inubushi, Y., Nagamine, K., Michine, Y., Ohashi, H., Yumoto, H., Yamauchi, K., Mimura, H., Kitamura, H., Katayama, T., Ishikawa, T. \& Yabashi, M. (2015). Nature. 524, 446-449. 